\title{
Rheumatological aspects of pathogenesis and treatment of COVID-19 infection
}

\author{
Desislava Kalinova, Rasho Rashkov \\ Clinic of Rheumatology, UMHAT St. Ivan Rilski University Hospital, Sofia, Bulgaria
}

\begin{abstract}
Contemporary rheumatology is a field dealing with the phenomena of autoimmune states and inflammation. Rheumatic diseases cover a wide spectrum of diseases of the musculoskeletal system, connective tissue and vessels. The occurrence of an immune, autoimmune and autoinflammatory response is often linked to different kinds of infections.

Which aspects of the coronavirus infection relate to rheumatological therapy and practice? In order to answer this question one needs to look at the pathogenesis of the SARS-CoV-2 infection. Antimalarial drugs may block antigen presentation of the viral peptides from antigen presenting cells, as they may alter the lysosomal proteases that mediate the viral entry in the cells and have demonstrated efficacy in improving the infection. Anti-IL-6 may interfere with cytokine storm in severe cases and use of tocilizumab has had good results in a small cohort. Baricitinib not only plays a role in inhibiting the synthesis of cytokines but it also has a function in suppressing receptor-mediated endocytosis.

The constantly new and tested concepts in the treatment of COVID testify to the growing knowledge about the virus, but also to the need for more targeted therapy. Treatment regimens have been developed for both patients with COVID-19 and those with symptomatic SARS-CoV infection and rheumatic disease. This article is an attempt to discuss the management of COVID-19 and coexisting rheumatic disease.
\end{abstract}

Key words: COVID-19 infection, anti-rheumatic drugs, rheumatic disease, pneumonitis.

\section{Introduction}

Rheumatology as a field deals with conditions of the musculoskeletal system, connective tissues, and vessels, and the majority of rheumatic diseases are closely linked with autoimmunity and the inflammatory state. The occurrence of an immune, autoimmune and autoinflammatory response is also often associated with different kinds of infections (viral or bacterial).

All infections come in three phases of development bacteraemia or viraemia, infectious-allergic, and immunological phase [1]:

1. Bacteraemia, viraemia - bacteria or the virus directly attack the organism and make it susceptible to other infections. This makes an infection in its initial stage a subject for infectious disease specialists and epidemiologists.

2. Infectious-allergic phase - a cytokine storm develops and causes damage due to the release of inflammatory cytokines and other inflammatory mediators.

3. Immunological phase - antibodies are created, which may (or may not) result in acquired immunity. In the course of various infections, often autoantibodies which target structures of the organism itself can also develop. This causes the inflammatory reaction from phase two to persist and morph into an autoimmune inflammation [1].

Rheumatology pertains to the infectious-allergic and immunological phases of the infectious process. These two pathogenic processes are the foundation of differ-

Address for correspondence:

Desislava Kalinova, Clinic of Rheumatology, UMHAT St. Ivan Rilski University Hospital, 13 Urvich St., 1612 Sofia, Bulgaria,

e-mail: d_kalinova666@abv.bg

Submitted: 7.05.2020; Accepted: 3.08.2020 
ent rheumatic diseases - inflammatory joint diseases (e.g. rheumatoid arthritis), systemic connective tissue diseases (systemic lupus erythematosus, progressive systemic sclerosis, vasculitis), inflammatory and autoinflammatory syndromes such as gout or periodic fever syndrome, and soft tissue inflammation (polymyalgia rheumatica). All of these conditions are linked to the antigen presentation of external antigens or autoantigens, the activation of the humoral and/or cell-mediated immune response, the activation of $B$ and $T$ lymphocytes, the synthesis of different cytokines (IL-1, IL-6, TNF- $\alpha$, IL-12, IL-18, GM-CSF, IFN- $\gamma$ ), and the synthesis of autoantibodies (different antinuclear antibodies, Abs against extractable nuclear antigens, antineutrophil cytoplasmic antibodies, antiphospholipid Abs, etc.).

In recent years, rheumatology has advanced significantly in treating the aforementioned diseases. This stems both from "cracking" the pathogenesis of rheumatic diseases on the one hand, and from the correctly combining disease-modifying anti-rheumatic drugs with new biologic therapy (monoclonal antibodies against different soluble cytokines - TNF- $\alpha$, IL-6, IL-1, receptor antagonist of TNF- $\alpha$, cluster blockers (anti-CD 20; rituximab), or kinase inhibitors (e.g. tofacitinib, baricitinib).

\section{Which aspects of the coronavirus infection relate to rheumatological therapy and practice?}

In order to answer this question one needs to look at the pathogenesis of the SARS-CoV-2 infection. Coronaviruses are enveloped viruses with a positive sense single-stranded RNA genome. Coronaviruses disease 2019 (CoViD-19) has emerged over the past months as a clinical syndrome caused by a novel beta-coronavirus, named severe acute respiratory syndrome (SARS)-CoV2 [2]. While the pathogenesis is not entirely narrowed down, it has been established that it involves several consecutive processes [2]:

\section{Coronavirus entry and replication}

All coronaviruses express a surface glycoprotein termed a "spike" which binds to the host receptor for viral entry that has been identified as angiotensin-converting enzyme 2 receptors (ACE2r) [3], expressed by mature lung epithelial cells, enterocytes, kidney proximal tubular cells and endothelial cells [4]. After receptor binding, lysosomal proteases cleave the spike protein, releasing the signal peptide that facilitates viral entry into the cells [5]. After the virus enters the cells, the viral RNA genome is released into the cytoplasm and is translated into two polyproteins and structural proteins, after which the viral genome begins to replicate [6].

\section{Antigen presentation in coronavirus infection}

While the virus enters the cells, its antigen will be presented to the antigen presentation cells, which is a central part of the body's anti-viral immunity. Antigenic peptides are presented by major histocompatibility complex (MHC) and then recognized by virus-specific cytotoxic $T$ lymphocytes. The antigen presentation of SARS-CoV mainly depends on MHC I molecules, but MHC II also contributes to its presentation [2].

\section{Humoral and cell-mediated immune response}

Antigen presentation subsequently stimulates the body's humoral and cellular immunity, which are mediated by virus-specific $B$ and $T$ cells [2].

\section{Cytokine storm in COVID-19}

The main mechanism for cytokine storm is an uncontrolled systemic inflammatory response resulting from the release of large amounts of pro-inflammatory cytokines (IFN- $\alpha$, IFN- $\gamma$, IL-1 $\beta$, IL-6, IL-12, IL-18, IL-33, TGF- $\beta$, etc.) and chemokines (CCL2, CCL3, CCL5, CXCL8, CXCL9, CXCL10, etc.) by immune effector cells (Li). The patients with severe SARS-CoV infection show elevated levels of IL-6, IFN- $\gamma$, and CCL5, CXCL8, CXCL10 in serum compared to those with the mild-moderate disease [7].

\section{Coronavirus immune evasion}

Thus, if one were once again to posit the question "Which aspects of the coronavirus infection relate to rheumatological therapy and practice?", the answer would be the antigen presentation of the pathogen from antigen-presenting cells (macrophages and dendritic cells) and the appearance of a cytokine storm, which leads to the development of pneumonitis in COVID-19 infections. The clinical symptoms and pathomorphological characteristics of the pneumonitis make it resemble acute respiratory distress syndrome.

Pneumonitis attacks the alveolocapillary membrane and disrupts pulmonary diffusion, which leads to respiratory failure. The pulmonary complications in human COVID-19 patients are due to an exuberant local inflammatory response with diffuse alveolar damage. Patients dying because of SARS have lung consolidation, oedema and mucopurulent material in the bronchial tree. On microscopic examination, alterations such as diffuse alveolar damage, hyaline membrane and fibrin formation, and neutrophil and macrophage infiltrates were detected in the interstitium and alveoli [8].

Similar pneumonites in rheumatology are: lupus pneumonitis, pneumonitis in ANCA-associated vasculi- 
tis (microscopic polyangiitis, granulomatosis with polyangiitis, eosinophilic granulomatosis with polyangiitis), pneumonitis in Goodpasture syndrome, interstitial lung disease with "ground-glass" opacities in progressive systemic sclerosis and myositis.

When pneumonitis has already occurred, the treatment is etiologic and pathogenic. In the case of pneumonitis or acute respiratory distress syndrome caused by COVID-19, it is essential to block, in order of importance, the "cytokine storm" and antigen presentation of the viral antigens from macrophages and dendritic cells to viral-specific T- and B-lymphocytes, which synthesize cytokines, chemokines and antibodies. The direct treatment of the viral infection is of course of paramount importance as well.

Therefore, from a rheumatological standpoint, the treatment of acute respiratory distress syndrome that developed due to COVID-19 entails the following steps:

1. Blocking antigen presentation from antigen presenting cells to T- and B-lymphocytes, which synthesize cytokine, chemokine and antibodies.

2. Managing the "cytokine storm" - it has been established that the leading cytokines are IFN- $\alpha$, IFN- $\gamma$, IL-1 $\beta$, IL-6, IL-12, IL-18, IL-33, TGF- $\beta$.

Cytokines and chemokines play a key role in the immune response against viral infections, and their altered production has been demonstrated in both SARS and MERS coronavirus infections. Such altered levels have been shown to be likely due to the low synthesis of antiviral cytokines such as interferons IFN- $\alpha$ or $\beta$ and in concert increased levels of other pro-inflammatory cytokines/chemokines that have pathogenic consequences. Among them, IL-1, IL- 6 and other pro-inflammatory cytokines were shown to be significantly more elevated in patients with severe compared to uncomplicated SARS or MERS infection $[9,10]$.

3. Attacking the virus directly with medication.

4. Attacking the virus directly with disinfectants and UV radiation.

5. Resuscitation measures if the applied therapeutic measures do not yield results.

It turns out that some of these goals can be attained with specific anti-rheumatic drugs.

Managing the "cytokine storm" can be implemented with the aid of glucocorticoids (medication with pronounced anti-inflammatory effect). Glucocorticoids are proven to be much more effective than non-steroidal anti-inflammatory drugs in treatments aimed at inhibiting prostaglandins, cytokines (TNFs, IFNs) and other inflammatory mediators. It is advised to apply glucocorticoids at a ratio of $1-2 \mathrm{mg} / \mathrm{kg}$. If necessary, a high dosage of these drugs can be used - $1 \mathrm{~g} /$ day, called pulse therapy [11].
Luckily, suppressing cytokine knots in the genesis of pneumonitis is possible. This can be done by applying biologic medication, monoclonal antibody, the antagonist of IL-6 - tocilizumab (RoActemra, Roche). Tocilizumab is a drug developed in Japan that has been successfully used in recent years as a treatment of rheumatoid arthritis. It has been shown that high plasma levels of IL-6 correlate with the severity and the prognosis of COVID-19 [12]. This explains the successful application of tocilizumab for pneumonitis that develops during the course of COVID-19 [13]. According to the published protocols for treating COVID-19 cases with severe pulmonary complications, tocilizumab is applied with a dose of $800 \mathrm{mg}$ i.v. [11]. IL-6 receptor blockers and corticosteroids at a daily dose of $1 \mathrm{mg} / \mathrm{kg} /$ day are good choice for ARDS and severe pneumonitis; otherwise they should be used at their lowest doses possible and should not be abruptly stopped.

Another possible medication with assumed potential effects on pneumonitis is baricitinib. Baricitinib is an inhibitor for Janus kinase 1 and 2 - enzymes which participate in intracellular signalling that leads to the synthesis of different cytokines. Baricitinib is applied within rheumatological practice as a treatment for rheumatoid arthritis. With regards to COVID-19, this JAK kinases inhibitor not only plays a role in inhibiting the synthesis of cytokines but it also has a function in suppressing receptor-mediated endocytosis [14, 15]. Although baricitinib has a potential role for the treatment of COVID-19, the National Institutes of Health $(\mathrm{NIH}$, USA) and the National Centre for Infectious Diseases of Singapore (NCID) do not recommend routine use of baricitinib outside a trial setting. Currently, several ongoing studies of baricitinib for COVID-19 are likely to report results in the months ahead, and their findings will prove whether it should be used more widely in this setting [15].

The blocking of antigen presentation of the viral peptides from antigen-presenting cells (macrophages, dendritic cells) can be achieved by two antimalarial drugs, chloroquine and hydroxychloroquine, both of them being quinine derivatives. The drugs' operating mechanism includes:

- inhibiting the proliferative response of T- and B-lymphocytes,

- suppressing the modification and presentation of the antigens,

- inhibiting the synthesis of IL-1 and TNF- $\alpha$ from macrophages, as well as prostaglandin E from lymphocytes,

- blocking the synthesis of IL-1, IL-6,

- suppressing the synthesis of IFN- $\gamma$ from lymphocytes,

- suppressing the levels of IL-6, as well as of CD8+ cells in patients with systemic lupus erythematosus. 
These qualities of antimalarial medications have been used in rheumatological practice for the treatment of arthritis and systemic connective tissue diseases. The primary therapeutic indication of these drugs is for the treatment of malaria. In the case of COVID-19, it has been established that chloroquine and hydroxychloroquine cut the pathogenetic chain off at its inception by blocking receptor-mediated endocytosis [16, 17]. Chloroquine and hydroxychloroquine alone (at a dose over $6.5 \mathrm{mg} / \mathrm{kg} /$ daily), or in combination with azithromycin have possible cardiotoxicity as a side effect, especially QT interval prolongation, rhythm and conduction disturbances [17]. Prior to as well as during the treatment it is necessary to perform ECG. If the patient has a cardiac co-morbidity, a combination of both medicines must be applied with caution, or not at all.

Finally, directly attacking the virus and subsequent infections is best achieved through the use of antiviral medications (remdesivir, lopinavir/ritonavir, oseltamivir) and antibiotics with good intracellular penetration (azithromycin) [11].

\section{Conclusions}

From a rheumatological standpoint, treatment for COVID-19 should encompass the following:

1. Coronavirus treatment: antiviral medications, antibiotics with good intracellular penetration (azithromycin) for 10 days [11].

2. Suppressing the antigen presentation: chloroquine and hydroxychloroquine. In particular, hydroxychloroquine is recommended to apply in a loading dose of $800 \mathrm{mg}$ followed by $400 \mathrm{mg}$ for 4 days $[16,17]$.

3. In the case of pneumonitis: glucocorticoids, the dose limit not exceeding 1-2 mg/kg i.v. a day. As far as pulse therapy with glucocorticoids is considered, the effect has not been proven [11].

4. In the case of severe pulmonary complication: tocilizumab (RoActemra) - monoclonal antibody against IL-6, administered in the dose of 4-8 $\mathrm{mg} / \mathrm{kg}$ i.v. at a recommended daily intake of $400 \mathrm{mg}$ i.v. The infusion should last for an hour and if there is no effect, the same dose, $400 \mathrm{mg}$ i.v., can be repeated in 12 hours. According to the available publications, $90 \%$ of patients recover within a number of days after tocilizumab treatment and pulmonary infiltrates regress [11].

5. Oxygen therapy.

Based on publication data, it is notable that if the patient does not suffer from a developed acute respiratory distress syndrome, the infection presents itself as either mild acute respiratory disease or is asymptomatic. However, should a pulmonary complication ensue, the treatment has to be as presented above.
Chloroquine and hydroxychloroquine have been used in rheumatological practice for about 30 years so far; glucocorticoids have been in use for over 40 years; tocilizumab has established itself for the treatment of some rheumatic conditions for more than 10 years. According to ACR Guidance for the management of adult patients with rheumatic diseases during the COVID-pandemic, regardless of COVID-19 severity, chloroquine/ hydroxychloroquine may be continued, but sulfasalazine, methotrexate, leflunomide, immunosuppressants, non-IL- 6 biologics, and JAK inhibitors should be stopped in a case of symptomatic SARS CoV-2 infection.

These positive results allow us to suggest the treatment scheme for COVID-19 related pulmonary complications which has been described above. Of course, it is based on the pathogenesis of the viral infections and the existing published data as well as the fact that it concerns a viral infection with a specific tropism towards particular bodily tissues and organs. The scientific research on SARS-CoV and its possible therapies are based on the knowledge of previous coronaviruses and on data recently made available by the Chinese scientific society.

The authors declare no conflict of interest.

\section{References}

1. Klimpel GR. Immune defenses. In: Medical microbiology, $4^{\text {th }}$ ed. NCBI Bookshelf; 1-21.

2. Li X, Geng M, Peng Y, et al. Molecular immune pathogenesis and diagnosis of COVID-19. J Pharm Analysis 2020; 10: 102108, DOI: 10.1016/j.jpha.2020.03.001.

3. Lu R, Zhao X, Li J, et al. Genomic characterization and epidemiology of 2019 novel coronavirus: implications for virus origins and receptor binding. Lancet 2020; 395: 565-574, DOI: 10.1016/S0140-6736(20)30251-8.

4. Chen J, Subbarao K. The immunobiology of SARS. Annu Rev Immunol 2007; 25: 443-472, DOI: 10.1146/annurev.immunol.25.022106.141706.

5. Zheng $\mathrm{Y}$, Shang J, Yang C, et al. Lysosomal proteases are a determinant of coronavirus tropism. J Virol 2018; 92: e01504-e01518, DOI: 10.1128/JVI.01504-18.

6. Perlman S, Netland J. Coronaviruses post-SARS: update on replication and pathogenesis. Nat Rev Microbiol 2009: 7: 439450, DOI: $10.1038 /$ nrmicro2147.

7. Min CK, Cheon S, Ha NY, et al. Comparative and kinetic analysis of viral shedding and immunological responses in MERS patients representing a broad spectrum of disease severity. Sci Rep 2016; 6: 25359, DOI: 10.1038/srep25539.

8. Ceribelli A, Motta F, De Santis M, et al. Recommendations for coronavirus infection in rheumatic diseases treated with biologic therapy. J Autoimmun 2020; 109: 102442, DOI: 10.1016/j.jaut.2020.102442. 
9. Channappanavar R, Perlman S. Pathogenic human coronavirus infections: causes and consequences of cytokine storm and immunopathology. Semin Immunopathol 2017; 39: 529-539, DOI: 10.1007/s00281-017-0629-x.

10. Yoshikawa T, Hill T, Li K, et al. Severe acute respiratory syndrome (SARS) coronavirus-induced lung epithelial cytokines exacerbate SARS pathogenesis by modulating intrinsic functions of monocyte-derived macrophages and dendritic cells. J Virol 2009; 83: 3039-3048, DOI: 10.1128/JVI.01792-08.

11. Diagnosis and treatment protocol for novel coronavirus pneumonia (trial version 7) (Released by National Health Commission \& State Administration of Tradiitional Chinese Medicine on March 3, 2020).

12. Le RQ, Li L, Yuan W, et al. FDA approval summary: tocilizumab for treatment of chimeric antigen receptor $T$ cell-induced severe of life-threatening cytokine release syndrome. Oncologist 2018; 23: 943-947, doi: 10.1634/theoncologist.2018-0028.

13. Xh Xu M, Li T, Sun W, et al. Effective treatment of severe COVID-19 patients with Tocilizumab. ChinaXiv 2020; 20200300026
14. Richardson P, Griffin I, Tucker C, et al. Baricitinib as potential treatment for 2019-nCoV acute respiratory disease. Lancet (London, England) 2020; 395: e30-e31, DOI: 10.1016/S01406736(20)30304-4.

15. Yang Y, Peng F, Wang R, et al. The deadly coronaviruses: the 2003 SARS pandemic and the 2020 novel coronavirus epidemic in China. J Autoimmun 2020; 109: 102434, DOI: 10.1016/j. jaut.2020.102434.

16. Gao J, Tian Z, Yang X. Breakthrough: Chloroquine phosphate has shown apparent efficacy in treatment of COVID-19 associated pneumonia in clinical studies. Biosci Trends 2020; 14 : 72-73, DOI: 10.5582/bst.2020.01047.

17. Yao X, Ye F, Zhang $M$, et al. In vitro antiviral activity and projection of optimized dosing design of hydroxychloroquine for the treatment of severe acute respiratory syndrome coronavirus 2 (SARS-CoV-2). Clin Infect Dis 2020; 71:732-739, DOI: 10.1093/ cid/ciaa237. 